\title{
Distributed Predictive Control Strategy for Frequency Restoration of Microgrids Considering Optimal Dispatch
}

\author{
Alex Navas F., Student Member, IEEE, Juan S. Gómez, Jacqueline Llanos, Member, IEEE, Erwin Rute, \\ Doris Sáez, Senior Member, IEEE, and Mark Sumner, Senior Member, IEEE
}

\begin{abstract}
Microgrids are the cornerstone for a new model of electrical generation based on renewable resources. Commonly microgrids are controlled with a centralised hierarchical structure, which is inherited from power systems. However, a timescale separation between traditional fast frequency restoration and slow economic dispatch may be counterproductive in the long run because the slow long-term economic dispatch increases the prediction uncertainty. In an effort to improve the economical operation of microgrids, this work proposes a distributed model predictive control strategy for the operation of isolated microgrids based on a consensus strategy that tackles both the economic dispatch and frequency restoration over the same timescale. The proposed controller can operate without knowledge of the microgrid's topology: instead, typical local measurements and other information from neighbouring generation units are required. Experimental results demonstrate that the controller is robust to load variations and communication issues, but the plug-and-play nature of the system is preserved.
\end{abstract}

Index Terms-Distributed predictive control, predictive optimal dispatch, Microgrids, cooperative control, frequency restoration.

\section{INTRODUCTION}

$\mathbf{M}$ ICROGRIDS (MGs) will be an essential feature of future power systems, as they enable the full integration of distributed energy resources (DERs). A MG is defined as "a group of interconnected loads and DERs within clearly defined electrical boundaries that acts as a single controllable entity with respect to the grid" [1]. MGs aim to achieve adequate real and reactive power sharing among distributed generation (DG) units, while frequency and voltage levels must be kept within safe levels [2]. MGs can operate in both gridconnected or island-mode. MGs that do not have a connection with the grid, because of technical, geographical or economic

Alex Navas F., and Erwin Rute are with the Department of Electrical Engineering, University of Chile, Santiago, Chile. Doris Sáez is with the Department of Electrical Engineering, University of Chile and Instituto Sistemas Complejos de Ingeniería (ISCI); emails: alex.navas@ug.uchile.cl, dsaez@ing.uchile.cl. Mark Sumner is with the PEMC, University of Nottingham, Nottingham, UK. Juan S. Gómez is with the Department of Electrical Engineering, Pontificia Universidad Católica de Chile, Santiago, Chile. Jacqueline Llanos is with the Departamento de Eléctrica, Electrónica y Telecomunicaciones, Universidad de las Fuerzas Armadas ESPE, 171-5-231B Sangolquí, Ecuador

This study was supported by the FONDECYT 1170683 grant, SERC-Chile ANID/FONDAP/15110019, ISCI ANID PIA/BASAL AFB180003, and PhD scholarships from ANID/Doctorado Nacional 2019-21190961 and from Secretaría de Educación Superior, Ciencia, Tecnología e Innovación de Ecuador, SENESCYT/ARSEQ-BEC-005848-2018. constraints are usually called isolated MGs [3]. Isolated MGs must be controlled with extreme care because the number of DGs available to tackle voltage and overloading problems is limited [3]. Isolated MGs must autonomously regulate voltage amplitude and frequency through the MG's control system. For most DERs, power electronics (PE) interfaces are needed to connect the DER to a MG. The sinusoidal rectifier is the most common configuration [4].

Typically, the control tasks of an isolated MG are split into three control levels, where each control level operates on a different time-scale [4]. The first level, which is the fastest of all (milliseconds), maintains the stability of the MG and ensures correct power-sharing [2]. This control level is comprised of inner current controllers, outer voltage controllers, and droop control loops; the latter allows variations of real/reactive power to be reflected as variations of frequency/voltage, as in large power systems [4]. Nevertheless, droop control produces deviations in frequency and voltage amplitude. At a slower time-scale (seconds) the setpoint values of frequency and voltage can be restored; the controllers used to manage this are known as the secondary control level [5]. The longterm tasks of a MG (seconds to minutes), such as optimal dispatch and coordination of MGs with the main grid, are performed by the tertiary control [6]. Most of the control solutions proposed in MGs have been derived from largescale electric power systems. However, as MGs possess low inertia due to the use of renewables and PE interfaces, the time-scale separation between secondary and tertiary control may be counterproductive [7], [8].

There are typically three architectures proposed for secondary control; these are centralised, decentralised and distributed [5]. Centralised control can give an optimal solution, but it has a common point of failure in the communication network. Decentralised controllers do not need a communication channel because each local controller takes actions based on their own measurements, but an optimal-solution is difficult to achieve [5]. Distributed controllers give a nearoptimal solution, and they are robust against communication failures [9].

Previously, proportional integral (PI) controllers were used at the secondary level [10]. However, thanks to advances in communication speeds and improved hardware capabilities of controllers, model predictive control (MPC) is being incorporated at the secondary control level, improving the overall performance of microgrids as these controllers are 
multi-objective and robust against communication delays [11], [12]. In distributed control, the consensus and cooperative control algorithms have attracted the attention of the MG community [9]. The multi-agent system (MAS) cooperative control method in particular aims to achieve system objectives cooperatively by mimicking the behaviour of biological phenomena [13].

Controllers based on MAS at the secondary level have been reported. For example, the authors of [10] proposed the distributed averaging proportional integral (DAPI) controller, which uses PI controllers for frequency and voltage restoration with an adjacency matrix that represents the communication network of the MG. The advantage of this controller is its distributed structure; however, real power is shared proportionally among DG units. The authors of [14] present a finitetime control approach to restore voltage and a DAPI controller to restore frequency; the former controller guarantees a fixed time convergence for voltage restoration and decouples the secondary controllers. A variation of the DAPI controller is presented in [15] where integral control actions are shared among the agents. The latter controller improves the transient of the proportional real power sharing among the DG units. However, as these controller are based on PI controllers, they neither include dynamic models of DGs nor include its physical power limits.

Recently, distributed control schemes based on MPC (DMPC) have been reported in MGs [11], [12], [16]-[18]. For instance, in [18], a non-linear DMPC is presented as an energy management system at the tertiary level for grid-connected microgrids, which is dependent on external load forecasts (and its inherent prediction uncertainty). DMPC at the secondary level has been reported in several publications. The authors of [11] present a feedback linearization DMPC for frequency and voltage restoration, considering the voltage and current at the LC filter output as state variables. The authors of [16] present a DMPC controller for voltage restoration, while frequency is restored using a variation of the DAPI controller with a finitetime observer. The authors of [17] present a DMPC controller based on a consensus version of the alternating direction method of the multipliers algorithm to regulate frequency in a networked MG system by manipulating the voltages of voltage-sensitive loads. The authors of [12] propose and validate experimentally a DMPC for frequency and voltage restoration using droop models and power transfer models; also real and reactive proportional power sharing is considered based on the concepts of [10]. The power-sharing is based on consensus over the real and reactive power contributions from each generation unit in the MG using an adjacency matrix. Using external measures this controller avoids the necessity to model the MG topology. Only [19] proposes a DMPC for economic dispatch and frequency restoration via simulation at the secondary level. This work includes both operation and maintenance costs within its formulation. However, it does not use consensus for the economic dispatch, and it assumes an ideal communication network. Nevertheless, none of the DMPC methods proposed for the secondary level take into account the overall economic operation of the MG via a consensus strategy.
There is a clear tendency to eliminate the time-gap separation between the secondary and tertiary control and to implement distributed controllers in isolated MGs [7], [10], [20]. In a distributed fashion [7], [21], [22] demonstrated that the economic dispatch can be integrated into the same timescale of frequency and voltage restoration. The authors of [21] reformulate the optimisation problem to achieve the KarushKuhn-Tucker (KKT) conditions of linear optimal power flow. Then, a control action from a PI controller is added to a droop controller to achieve a near-optimal economic dispatch. The authors of [22] achieved real power and reactive power dispatch including voltage and frequency regulation in a decomposition of the optimisation problem, but only simulation results are provided. As the previously described distributed controllers only use PI controllers and rely on a fixed control law, they might not guarantee an optimal solution, as they do not consider real-time changes in the operation of the $\mathrm{MG}$ [23]. Furthermore, most studies are based on decomposition techniques [21], [22], [24] instead of solving an optimisation problem.

To the best of the authors' knowledge, the problem of combined frequency restoration and optimal dispatch in isolated MGs using DMPC has not been thoroughly studied in the existing literature. Furthermore, in most studies, maximum power constraints are not taken into account: these constraints can prevent equipment damage. In this paper, a distributed cooperative scheme for controllers is adopted based on MPC. Each controller seeks to dispatch its controllable unit based on its generation cost and to restore the microgrid's frequency deviation. The performance of the proposed controller is validated in a experimental microgrid. The case study for this work is a multi-nodal ac MG with three DERs, where each DG unit is emulated as a controllable generator with its own specific characteristics.

The contributions of this paper are as follows:

i A novel DMPC control is proposed for optimal dispatch and frequency restoration where both use the same timescale.

ii The proposed controller neither requires the modelling of the full MG nor the modelling of the connected loads. Furthermore, this controller operates with the same typical measurements used at the primary control level; thus, the number of physical measurements is reduced when compared with [12].

iii The proposed DMPC with economic dispatch scheme addresses communication delays, loss of communications and plug-and-play scenarios, unlike centralised MPC schemes.

iv The DMPC includes as equality constraints the droop, the real power transfer, and the phase angle equations to predict the behaviour of each DG. Additionally, terminal values and inequality constraints contribute to bound the feasible solution space. This reduces the optimisation time and enables the control strategy to be implemented in real-time controllers. Nevertheless, a stability analysis is out of the scope of this paper.

This paper is organised as follows. In Section II, the 
theoretical background of the controller is detailed. In Section Section III, the proposed DMPC controller is explained. Section IV presents the experimental setup and the experimental results obtained. Section V highlights the controller improvements with previous approaches. Finally, the conclusions are presented in Section VI.

\section{BACKGROUND}

In this section, the isolated ac MGs' framework employed for the optimal dispatch and frequency restoration is specified. First, the formulation of the centralised optimal dispatch is presented. Then, its extension for the distributed scheme is created.

\section{A. Centralised Economic Dispatch}

Consider a three-phase balanced $\mathrm{MG}$ with a set of $\mathbb{P}$ DGs, where $\mathbb{P}=\{1, \ldots, p\}$. The optimal dispatch establishes the least-cost dispatch of the controllable DG units, whilst ensuring that the total load is met. The optimal dispatch formulation is presented in (1a) and (1b). $\mathbb{P}$ is the set of DGs in the MG, $P_{i}$ is the real power contribution of DG $i$, $\mathbb{C}_{i}\left(P_{i}\right)$ is a convex cost function, $P_{D}$ is the total MG load, and $\mathbf{P}=\left\{P_{i}: i \in \mathbb{P}\right\}$. The traditional centralised economic dispatch (1) depends on a unique central controller, where its failure could compromise the economic dispatch of the microgrid. Therefore, a distributed control is a good alternative for higher reliability and security for the microgrid's operation [25].

$$
\begin{array}{ll}
\underset{\mathbf{P}}{\operatorname{minimise}} & \sum_{i=1}^{p} \mathbb{C}_{i}\left(P_{i}\right) \\
\text { subject to } & \sum_{i=1}^{p} P_{i}=P_{D}
\end{array}
$$

Assuming strong duality holds e.g. Slater's constraint qualification condition holds, the problem may be expressed through its Lagrange dual [26]. The Lagrangian function of the optimal dispatch problem is:

$$
\mathbb{L}\left(P_{i}, \eta\right)=\sum_{i=1}^{p} \mathbb{C}_{i}\left(P_{i}\right)+\eta\left(P_{D}-\sum_{i=1}^{p} P_{i}\right)
$$

Where the Lagrange multiplier $\eta$ is associated with the power balance constraint. The KKT stationary condition for the problem is defined in (3a). From (3a) it is possible to establish that at the optimal point, it must apply that the incremental cost (IC) function as defined by (3b).

$\frac{\partial \mathbb{L}}{\partial P_{i}}=\nabla \mathbb{C}_{i}\left(P_{i}\right)-\eta=0 \quad i \in \mathbb{P}$

$\eta=\nabla \mathbb{C}_{i}\left(P_{i}\right) \quad i \in \mathbb{P}$

The generation cost function for the i-th DG is stated by (4a) [8], where $a_{i}, b_{i}$ and $c_{i}$ are cost parameters defined in Section IV-B, and $P_{i}$ is its real power contribution. Furthermore, its IC is given by (4b).

$$
\begin{aligned}
& \mathbb{C}_{i}\left(P_{i}\right)=a_{i} P_{i}^{2}+b_{i} P_{i}+c_{i} \\
& \eta_{i}\left(P_{i}\right)=2 a_{i} P_{i}+b_{i}
\end{aligned}
$$

The economic dispatch problem redistributes the power contribution of all DGs to reach the same $\eta$ (3b) (KKT stationary condition), where $\eta$ corresponds to the (unique) dual variable associated with the demand-supply balance equation (1b) of the microgrid's optimal dispatch problem. Therefore, a distributed cooperative scheme can be designed that ensures the condition of $\eta_{i}=\eta_{j}=\eta$ in steady state, where $\eta_{j}$ is the IC of neighbouring DGs. Note that this distributed scheme intrinsically meets the demand-supply balance.

Based on the IC, a new distributed predictive cooperative control strategy is designed, aiming to provide an economic dispatch of real power while the frequency is regulated. This DMPC control strategy is based on the work presented in [12]. It is worth noting that in [12] the optimal economic dispatch is not considered. Moreover, the coupling between voltage regulation and reactive power sharing are not considered in the DMPC formulation. However, the DAPI controller reported in [10] is used to solve the voltage restoration and reactive power sharing.

\section{B. Distributed Model Predictive Control Principles}

MPC has been widely applied in microgrids because it is a multi-variable controller, which employs a model of the process and allows the inclusion of an object function and constraints in its formulation [23]. MPC solves an optimisation problem at each sample time by calculating a control action sequence to achieve the problem objectives; however, only the first control action is applied to the system and the optimisation problem is solved again at each next sample time (rolling horizon). Although centralised MPC gives a global solution to a problem, it has the main disadvantage of requiring a large communication structure, which could fail and compromise the operation of the entire system [12]. Furthermore, it presents a high computational burden, as the computational burden increases exponentially with the number of optimisation variables [23]. For these reasons, centralised MPC may be impractical to use at the microgrids' secondary control level. On the other hand, DMPC works on the basis of solving local optimisation problems, considering shared information from neighbours. The information exchange between controllers allows DMPC to achieve a similar solution to the centralised approach. However, DMPC reduces the computational burden and is more robust when failures occur.

DMPC eases the scalability of microgrids and preserves the plug-and-play capability, as no changes in the programming are needed when DGs are connected/disconnected. Related DMPC approaches at the secondary level [11], [12], [16], [17] were discussed in the introduction. However, none of these publications consider the inclusion of economic dispatch at the secondary level.

\section{Proposed Distributed Control Scheme}

The proposed controller neither depends on the MG's electrical topology nor on adjacent physical measurements i.e. only the typical measurements at the LC-output filter and voltage observers are needed. Therefore, the number of buses and distribution lines are irrelevant. To avoid modelling 
the entire MG, small coupling inductances $\left(L_{i}\right)$ are needed between the LC output-filter and the MG's connection node, as shown in the electrical configuration of Fig. 1. However, these inductances can be substituted for virtual impedance controllers [27].

To predict the power contribution of each DG, the phase angle deviation $\left(\delta \theta_{i}\right)$ between the local unit and the MG is determined [28]. For this, the voltage measurement $\left(V_{i}\right)$ at the output of the LC filter is used, then using a phase locked loop (PLL), its frequency $\left(\omega_{i}\right)$ and phase angle $\left(\theta_{i}\right)$ are estimated. In addition, the voltage $\left(\hat{V}_{i}^{B}\right)$ at the connection bar node (after coupling inductances $L_{i}$ ) is estimated by virtual meters based on non-linear reduced-order observers to reduce the hardware, while the performance of the controller is maintained. Then, with a PLL, its frequency $\left(\hat{\omega}_{i}^{B}\right)$ and phase angle $\left(\hat{\theta}_{i}^{B}\right)$ are estimated, as shown in Fig. 1.

The voltage observer is based on [29], whose main advantage is its linear dynamic for estimation error. Therefore, the observer gains can be tuned via pole placement, improving the transient performance of the observer and the convergence rate of the estimation error. It also has only a low computational burden. The basis of the observer is detailed as follows. The observer works in the $\alpha-\beta$ framework. The estimated states are defined by $\hat{V}_{\alpha, i}^{B}=V_{m} \sin \left(\theta_{i}\right)$ and $\hat{V}_{\beta, i}^{B}=V_{m} \cos \left(\theta_{i}\right)$, where $V_{m}$ depends on the $a b c-\alpha \beta$ transformation used. The observer's state space formulation is obtained from Kirchhoff's voltage law, whereas its inputs are the measured values of $V_{i}$ and $i_{i}$ (at the LC filter output), both in the $\alpha-\beta$ framework. A detailed explanation of the development of the voltage observer is presented in Appendix A.

The controller scheme for each DG is depicted in Fig. 1. Each DG is configured as a voltage source inverter (VSI) with its respective LC output-filter. Two control layers are highlighted. The primary control level is made up of $\omega-P$ and $V-Q$ droop controllers, outer voltage and inner current controllers. These controllers are on the direct-quadrature framework. The voltage observer also operates at this control level.

At the secondary level, the economic dispatch and frequency restoration controller is presented. This controller receives as inputs the local measurements/estimates $\left(P_{i}(k), V_{i}(k), \omega_{i}(k), \theta_{i}(k), \hat{V}_{i}^{B}(k), \hat{\omega}_{i}^{B}(k), \hat{\theta}_{i}^{B}(k)\right)$ of the ith-DG unit and the results of the optimisation problems of communicated neighbouring units. The controller has two outputs, which are the control actions' variations (vector $\left.\Delta \omega_{s, i}\right)$ and the results of the local optimisation problem $\mathbb{Y}_{i}$ (predicted values), both defined in Section III-C. Whereas the former passes through a discrete integrator to ensure zero error in steady-state, the latter is sent via the communication network. Note that both objectives of our proposal (frequency restoration and economic dispatch) are achieved with the same control action $\Delta \omega_{s, i}$.

\section{Communication Structure}

To share information among neighbouring DGs, the DMPC controller uses a full-duplex communication network. The proposed use of this network considers both latency and connectivity issues. Whereas the former expresses the time

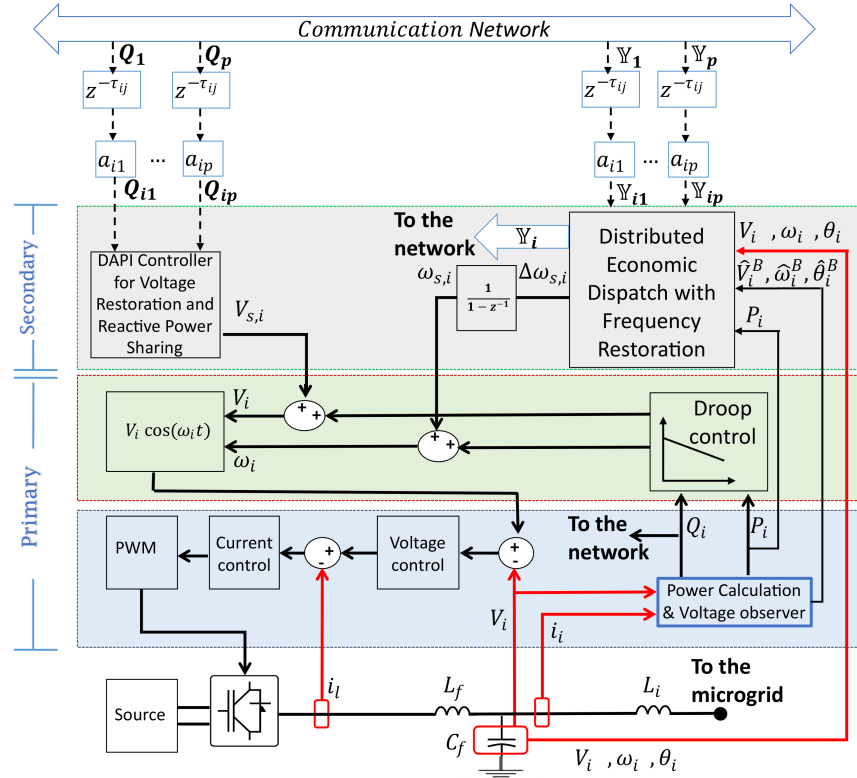

Fig. 1. Distributed optimal dispatch with frequency restoration for $D G_{i}$

interval $\left(\tau_{i j}\right)$ for a data packet to reach its destination, the latter reflects the communication topology between the DGs. Delays $\tau_{i j}$ are represented in sampling periods, and $\tau_{i j}=\tau_{j i}$. The bidirectional connectivity from/to $D G_{i}$ to/from $D G_{j}$ is defined by the adjacency term $a_{i j}$ (5) of the non-negative weighted adjacency matrix $A$ (defined in Section IV). As the communication is bidirectional, the associated graph is undirected; thus, $a_{i j}=a_{j i}$ [30].

$a_{i j}(k)= \begin{cases}1 & \text { Data from } \mathrm{DG}_{j} \text { arrives at } \mathrm{DG}_{i} \text { at } k \\ 0 & \text { Data from } \mathrm{DG}_{j} \text { does not arrive at } \mathrm{DG}_{i} \text { at } k \\ 0 & j=i\end{cases}$

\section{E. Dynamic Models}

As the proposed controller considers the real operation of a MG, the models that govern the dynamics of the DGs that make up the MG are included. These models are presented in (6). The droop model for frequency - real power $(\omega(t)-P(t))$ for the local ith-DG is presented in (6a). This relationship allows the DGs to interpret real power changes in the MG by producing a frequency deviation; furthermore, through this model, it is possible to connect the primary and secondary control levels. $\omega_{0}$ is the nominal frequency, $M_{p \omega, i}$ is the droop slope and $\omega_{s, i}(t)$ is the control action from the DMPC controller.

To determine the power transferred from the ith-DG to the MG, the phase angle difference must be determined. The phase angle difference $\left(\delta \theta_{i}\right)$ between the local DG unit and the MG through a coupling inductance $\left(L_{i}\right)$ is defined in (6b) [28], where $\theta_{i}, \omega_{i}$ are the phase angle and frequency at the LCoutput filter, and $\hat{\theta}_{i}^{B}$ and $\hat{\omega}_{i}^{B}$ are the phase angle and frequency after the coupling inductance, respectively.

The power transfer equation (6c) is included to govern the real power contribution from each DG unit to the MG. Where $V_{\mathrm{i}}(t)$ and $\hat{V}_{\mathrm{i}}^{B}(t)$ are the voltages before and after the coupling inductance $L_{i}$, and $B_{i}=1 /\left(L_{i} \cdot \omega_{0}\right)$ [28]. The use of an 
admittance matrix is avoided with this formulation, as this reduces the plug-and-play capability of DG units.

$$
\begin{aligned}
& \omega_{i}(t)=\omega_{0}+M_{p \omega, i} P_{i}(t)+\omega_{s, i}(t) \\
& \delta \theta_{i}(t)=\theta_{i}(t)-\hat{\theta}_{i}^{B}(t)=\int_{0}^{t}\left[\omega_{i}(\tau)-\hat{\omega}_{i}^{B}(\tau)\right] d \tau \\
& P_{i}(t)=B_{i} V_{i}(t) \hat{V}_{i}^{B}(t) \sin \left(\delta \theta_{i}(t)\right)
\end{aligned}
$$

\section{F. Discrete Time Models}

Prior to defining the predictive models, a discretization of the models of (6) is needed. These models are discretized using the forward Euler method, where $k=n T_{s e c}, n \in \mathbb{Z}^{+}$, and $T_{\text {sec }}$ is the sample time of the controller. As integrators are placed at the output of the predictive controllers to ensure zero error in steady-state (See Fig. 1) [23], the incremental operator $\left({ }_{\Delta} f(k)=[f(k)-f(k-1)]\right)$ is applied to (6a); thus, the optimisation problem is expressed in function of the control action's variation $\left(\Delta \omega_{s, i}\right)$. Additionally, a Taylor expansion is applied around the measured/estimated point $\left\{\omega_{i}(k), \hat{\omega}_{i}^{B}(k)\right.$, $\left.V_{i}(k), \hat{V}_{i}^{B}(k), \delta \theta_{i}(k), P_{i}(k)\right\}$ to linearize the power transfer model (6c). The resulting linear discrete models are shown in (7c).

$$
\begin{aligned}
& \omega_{i}(k+1)=\omega_{i}(k)+M_{p \omega, i}\left[P_{i}(k+1)-P_{i}(k)\right]+\Delta \omega_{s, i}(k) \\
& \delta \theta_{i}(k+1)=\delta \theta_{i}(k)+T_{s e c}\left[\omega_{i}(k+1)-\hat{\omega}_{i}^{B}(k)\right] \\
& P_{i}(k+1)=P_{i}(k) \\
& \quad+\left[\delta \theta_{i}(k+1)-\delta \theta_{i}(k)\right] B_{i} V_{i}(k) \hat{V}_{i}^{B}(k) \cos \left(\delta \theta_{i}(k)\right)
\end{aligned}
$$

\section{Proposed Distributed Predictive CONTROL}

Unlike [12], where the aim was that all generation units contribute to the power-sharing of real power in proportion to their maximum capacity, in this work a more realistic formulation is proposed that meets the economic dispatch, where the most economical generation units are the ones that contribute most to the power-sharing as long as power limits are not exceeded.

MPC uses the discrete-time model of the system, presented in (7), to predict the behaviour of the MG over a prediction horizon $\left(N_{y}\right)$, and a sequence of control actions $\left(N_{u}\right)$ is calculated through a numerical optimisation problem that minimises a cost function. The variables of both the prediction horizon and control horizon are contained in the vector $\mathbb{X}_{i}$ (defined in Section III-C ), which is the solution to the optimisation problem. Only the first control action is applied to the system, and the optimal control problem is repeated at each sample time with updated measures[23]. A challenge in the implementation of MPC controllers at the secondary control level is the definition of an optimisation problem with a low computational burden which can be solved in a short sample period [12]. The optimisation problem and how it is solved is detailed in the next section.

\section{A. Problem Statement}

To predict the response of the ith-DG at $k+m$ steps, where $m \in \mathbb{Z}^{+}$, the set of equations of (7) is generalised in (8). Note that although the coefficients produced in the linearization are updated each sample time, they are constant during the optimisation.

$$
\begin{aligned}
& \omega_{i}(k+m)=\omega_{i}(k+m-1)+M_{p \omega, i}\left[P_{i}(k+m)-P_{i}(k+m-1)\right] \\
& \quad+\Delta \omega_{s, i}(k+m-1) \\
& \delta \theta_{i}(k+m)=\delta \theta_{i}(k+m-1)+T_{s e c}\left[\omega_{i}(k+m)-\hat{\omega}_{i}^{B}(k)\right] \\
& P_{i}(k+m)=P_{i}(k) \\
& \quad+\left[\delta \theta_{i}(k+m)-\delta \theta_{i}(k)\right] B_{i} V_{i}(k) \hat{V}_{i}^{B}(k) \cos \left(\delta \theta_{i}(k)\right)
\end{aligned}
$$

A set of operational constraints are included in the MPC formulation. These are equality constraints to ensure appropriate performance of the controller and inequality constraints to guarantee the solution is within the physical power capacity of each DG.

Equation (9a) represents a local average frequency. This average is calculated with only the information communicated from the other DGs, which is determined by the adjacency term $a_{i j}$. Additionally, the term $\hat{\tau}_{i j}$ represents the timedelay estimation. These two terms provide robustness against communication failures and latency effects, respectively. The terminal constraint ( $9 b$ ) is included to guarantee convergence of the distributed predictive scheme to the tracking value (nominal frequency) at the end of the prediction horizon $N_{y}$ [31].

$\bar{\omega}_{i}(k+m)=\frac{\omega_{i}(k+m)+\sum_{j=1}^{P} a_{i j}(k) \omega_{j}\left(k+m-\hat{\tau}_{i j}\right)}{1+\sum_{j=1}^{P} a_{i j}(k)}$

$\bar{\omega}_{i}\left(k+N_{y}\right)=\omega_{0}$

The generalisation of the IC for the prediction horizon is expressed in (10). This term is used in the objective function of our controller, as shown in (12).

$\eta_{i}(k+m)=2 a_{i} P_{i}(k+m)+b_{i}$

Finally, the real power contribution of the ith-DG is limited to its physical capacity through the inequality constraint (11); hence, the space solution is bound.

$P_{i \text { min }}(k) \leq P_{i}(k+m) \leq P_{i \max }(k)$

\section{B. Cost Function}

The multiobjective cost function is stated in (12) and is composed of three weighted terms, where each term seeks a specific objective. The first term represents the average frequency restoration, which is calculated only with the information communicated from other DGs. The second term penalises the control action sequence required to carry out at the same time both the regulation and consensus objectives; 
furthermore, the overshoot and settling time are adjusted with this term. The third term achieves the economic dispatch through a cooperative consensus over the predicted ICs between the local DG and the neighbouring (communicating) DGs. Therefore, the condition $\eta_{i}=\eta_{j}=\eta$ in steady state is enforced within the objective function. The terms $\lambda_{1 i}, \lambda_{2 i}$ and $\lambda_{3 i}$ are the tuning parameters, and $\hat{\tau}_{i j}$ is the delay estimation, which is considered as one sample period on the secondary level.

$$
\begin{aligned}
J_{i}(k) & =\sum_{m=1}^{N_{y}} \lambda_{1 i}\left(\bar{\omega}_{i}(k+m)-\omega_{0}\right)^{2} \\
& +\sum_{m=1}^{N_{u}} \lambda_{2 i}\left(\Delta \omega_{s, i}(k+m-1)\right)^{2} \\
& +\sum_{j=1, j \neq i}^{P} \sum_{m=1}^{N_{y}} \lambda_{3 i} a_{i j}(k)\left(\eta_{i}(k+m)-\eta_{j}\left(k+m-\hat{\tau}_{i j}\right)\right)^{2}
\end{aligned}
$$

\section{Quadratic Programming Formulation}

As the controllers considered have convex cost functions and linear constraints, they are solved using quadratic programming (QP). The cost function (12) with its respective equality and inequality constraints (8)-(11) are included in a QP formulation (13) via the matrices/vectors $H_{i}, A_{i}, B_{i}, A_{e q, i}, B_{e q, i}, F_{i}$. The output of the QP problem is in (14), where the set of predicted variables is contained in $\mathbb{X}_{p, i}$ (15) and the optimal control sequence is represented in $\mathbb{X}_{\Delta, i}(16)$.

$$
\begin{aligned}
& \underset{\mathbb{X}_{i}}{\operatorname{minimize}} \quad J_{i}(k):=\frac{1}{2} \mathbb{X}_{i}^{T} H_{i} \mathbb{X}_{i}+F_{i}^{T} \mathbb{X}_{i} \\
& \text { subject to } \quad A_{i} \mathbb{X}_{i} \leq B_{i} \\
& A_{e q, i} \mathbb{X}_{i}=B_{e q, i} \\
& \mathbb{X}_{i}=\left\{\mathbb{X}_{p, i}, \mathbb{X}_{\Delta, i}\right\} \\
& \mathbb{X}_{p, i}=\left\{\bar{\omega}_{i}(k+m), \omega_{i}(k+m), \delta \theta_{i}(k+m),\right. \\
& \left.P_{i}(k+m), \eta_{i}(k+m)\right\}_{m=1}^{N_{y}} \\
& \mathbb{X}_{\Delta, i}=\left\{\Delta \omega_{s, i}(k+m-1)\right\}_{m=1}^{N_{u}}
\end{aligned}
$$

Following the principle of MPC, only the first predicted control action of $\mathbb{X}_{\Delta, i}$ is applied in the frequency-real power droop controller. The optimisation is then repeated each sample time with updated measurements/estimations. To reduce the traffic over the communication network only the necessary predictions to achieve the cooperative objectives are shared $\mathbb{Y}_{i}$ (17).

$$
\mathbb{Y}_{i}=\left[\omega_{i}(k+m), \eta_{i}(k+m)\right]_{m=1}^{N y}
$$

To compute the QP problem (13), the QPKWIK algorithm is used, which is a stable variation of the classic active-set method [32]. This solver is able to generate $C_{++}$code to run on the experimental setup. The methodology to solve the DMPC scheme is described in Algorithm 1, which details all the necessary steps to obtain a cooperative solution.

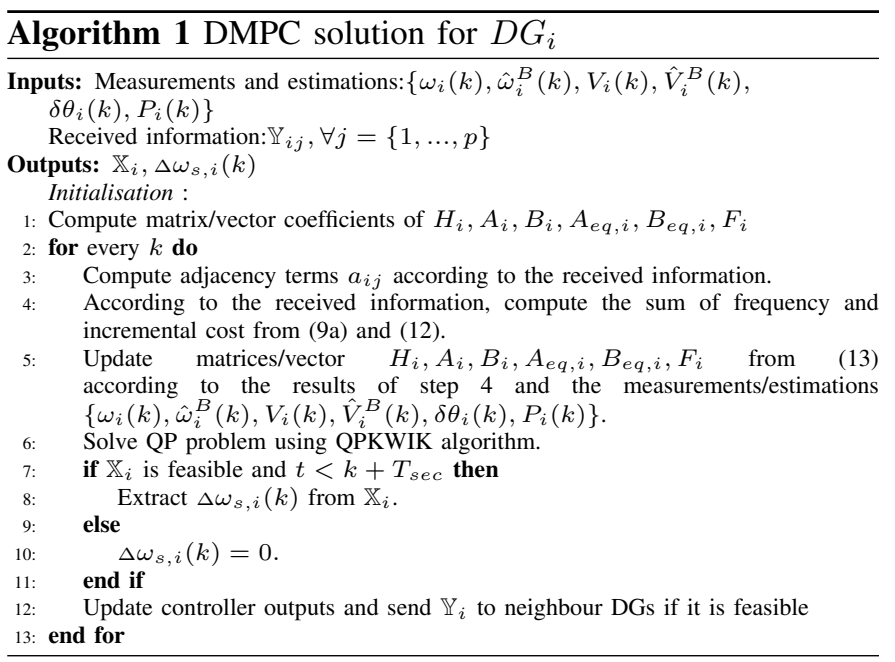

\section{EXPERIMENTAL SETUP AND RESULTS}

\section{A. Experimental MG Configuration}

The performance of the DMPC control strategy is assessed in a case study using the experimental MG configuration illustrated in Fig. 2a; this experimental MG was built in the MGs Control Lab of the University of Chile. The MG comprises three DG units, which are emulated by the PM15F120(DG 1 and DG2) and PM5F60(DG3) Triphase ${ }^{\circledR}$, as shown in Fig. 2 b. Each DG unit is controlled by a real-time target (RTT) computer, in which the DMPC control algorithm is uploaded. The minimum estimated delay corresponds to one sample period at the secondary level sample time. Table I and Table II present the droop parameters and electrical parameters, respectively.

\section{B. DMPC Design Parameters and Test Scenarios}

Table III presents the DMPC design parameters and weighting factors. All the design parameters were selected aiming to reduce the overall computational burden. This is because
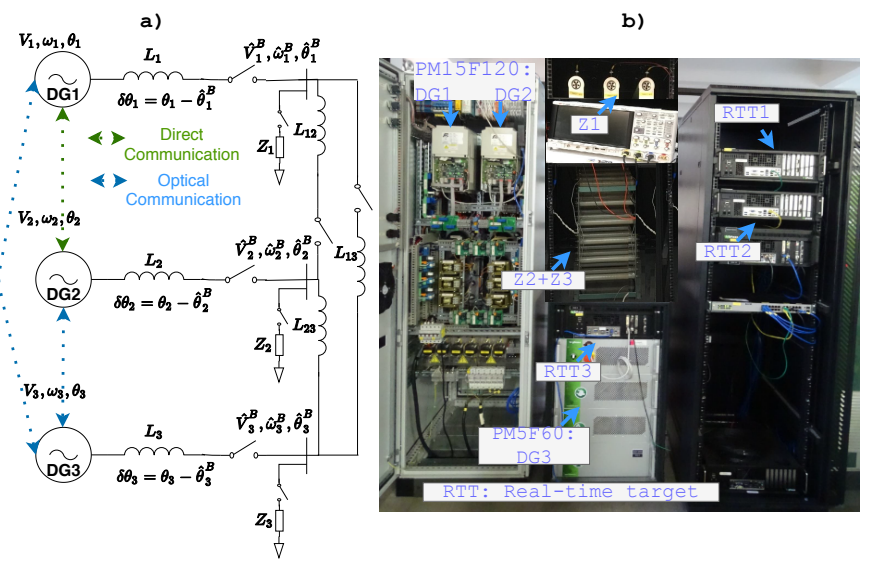

Fig. 2. a) MG Diagram, b) Experimental MG setup 
TABLE I

Power Capacities AND Droop Slopes

\begin{tabular}{l|l|l}
\hline \hline Parameter & Description & DG1-DG3 \\
\hline$P^{\max }[\mathrm{Kw}]$ & Maximum power capacity & 2.1 \\
\hline$M_{p \omega} \frac{r a d}{s W}$ & $P-\omega$ droop coefficients & $-2.38 \mathrm{E}-4$ \\
\hline$M_{q v} \frac{V}{V A R}$ & $Q-V$ droop coefficients & $-4.8 \mathrm{E}-3$ \\
\hline \hline
\end{tabular}

TABLE II

MG ELECTRICAL PARAMETERS

\begin{tabular}{l|l|l}
\hline \hline Parameter & Description & Value \\
\hline$T_{\text {prim }}[\mathrm{s}]$ & Primary level sample period & $1 / 16 \mathrm{E} 3$ \\
\hline$Z_{1}[\Omega]$ & Load 1 & $35(0.64 \mathrm{KW})$ \\
\hline$Z_{2}[\Omega]$ & Load 2 & $15(1.5 \mathrm{KW})$ \\
\hline$Z_{3}[\Omega]$ & Load 3 & $22(1.02 \mathrm{KW})$ \\
\hline$L_{i}[\mathrm{mH}]$ & Coupling inductance & 2.5 \\
\hline$L_{i j}[\mathrm{mH}]$ & Distribution line inductance & 2.5 \\
\hline$\omega_{0}[\mathrm{rad} / \mathrm{s}]$ & Nominal frequency & $100 \pi$ \\
\hline$V_{0}[\mathrm{~V}]$ & Nominal voltage (peak) & 150 \\
\hline$\omega_{c}[\mathrm{rad} / \mathrm{s}]$ & Droop controller cutoff frequency & $2 \pi$ \\
\hline \hline
\end{tabular}

TABLE III

CONTROLLER PARAMETERS AND WEIGHTS

\begin{tabular}{l|l|l}
\hline \hline Parameter & Description & Value \\
\hline$T_{s e c}[\mathrm{~s}]$ & Controller sample time & 0.05 \\
\hline$\hat{\tau}_{i j}[\mathrm{~s}]$ & Estimated communication delay & 0.05 \\
\hline$N y$ & Prediction horizon & 5 \\
\hline$N u$ & Control horizon & 5 \\
\hline$\lambda_{1 i}\left[\left(\frac{s}{\mathrm{rad}}\right)^{2}\right]$ & Average frequency error & $18.5 \mathrm{E} 2$ \\
\hline$\lambda_{2 i}\left[\left(\frac{s}{\mathrm{rad} d}\right)^{2}\right]$ & Frequency control action & $4.7 \mathrm{E} 4$ \\
\hline$\lambda_{3 i}\left[\left(\frac{1}{W}\right)^{2}\right]$ & Real power dispatch & $1.01 \mathrm{E}-4$ \\
\hline \hline
\end{tabular}

TABLE IV

DG COST PARAMETERS

\begin{tabular}{l|lll}
\hline \hline Parameter & DG1 & DG2 & DG3 \\
\hline $\mathrm{a}\left[\$ / k W^{2}\right]$ & 0.264 & 0.4 & 0.5 \\
\hline $\mathrm{b}[\$ / k W]$ & 0.067 & 0.1 & 0.125 \\
\hline $\mathrm{c}[\$]$ & 0 & 0 & 0 \\
\hline
\end{tabular}

the computational burden increases significatively with the sample time, and the prediction and control horizons [31]. While the sample time was selected considering the frequency open loop rise time $\left(T_{r}=0.7 \mathrm{~s}\right)$ as $T_{\text {sec }}=0.7 / 14=0.05 \mathrm{~s}$ [33], the prediction and control horizons were selected as 5 samples because with these values, the traffic over the communication network is reduced. The weighting factors were tuned heuristically looking for a trade-off between the control objectives. Table IV presents the generation costs for each DG unit taken from [21].

The controller was tested under four scenarios using the experimental MG. The first scenario presents the DMPC's performance when the MG experiences a load change. The second scenario shows the behaviour of the MG when there are latency effects over the communication network. The third
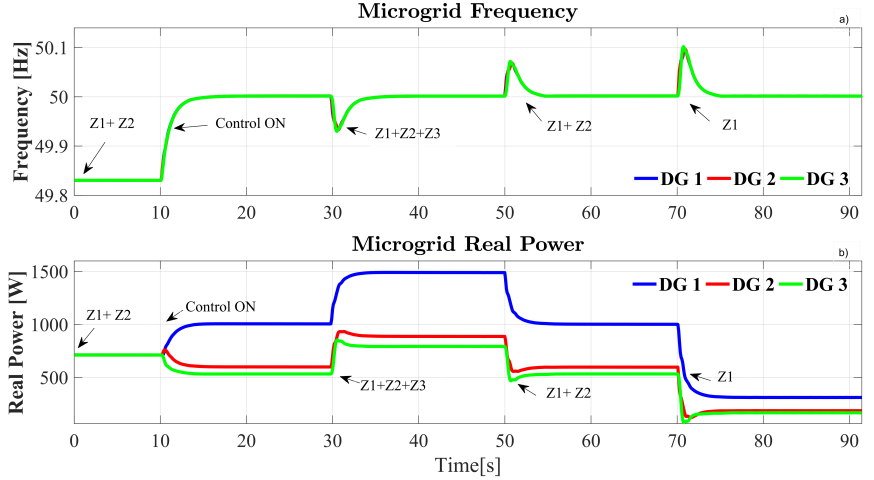

Fig. 3. a) Frequency regulation for load changes - Base Case, b) Real power injection for load changes - Base Case

scenario shows the effects of a failure in the communication network. Finally, the last scenario validates the Plug-andPlay capability, where DG2 is disconnected and reconnected from/to the MG. As voltage restoration and reactive power sharing are not the focus of this paper, their results are presented just for the base case.

\section{Scenario I (Base Case) - Load Changes}

This scenario presents the performance of the proposed controller in the MG when there are several load impacts. During the whole test, the adjacency matrix is not changed and is represented in (18).

$$
A(k)=\left[\begin{array}{lll}
a_{11} & a_{12} & a_{13} \\
a_{21} & a_{22} & a_{23} \\
a_{31} & a_{32} & a_{33}
\end{array}\right]=\left[\begin{array}{lll}
0 & 1 & 1 \\
1 & 0 & 1 \\
1 & 1 & 0
\end{array}\right]
$$

The test starts with the primary control enabled (internal loops and droop control) and two loads connected $\left(Z_{1}\right.$ and $\left.Z_{2}\right)$ at different nodes to observe that without the DMPC controller, the DG units share real power equally (see Fig. 3b before $10 \mathrm{~s}$ ) and the frequency deviates from its nominal value (see Fig. 3a before $10 \mathrm{~s}$ ). At $t=10 \mathrm{~s}$, the secondary controller is enabled, so the frequency is restored to its nominal value, as observed in Fig. 3a; and the real power is redistributed, as observed in Fig. 3b. Once the controller is enabled the real power is redispatched according to the DG's operating costs. As DG1 is the least expensive unit, it takes the majority of the load followed by DG2, which has an intermediate cost. Furthermore, DG3 takes the lowest the load as it is the most expensive. At 30 seconds, the MG is subjected to its total load $\left(Z_{3}\right.$ is connected). Finally, the loads $Z_{3}$ and $Z_{2}$ are disconnected at $t=50$ and $t=70$, respectively. Fig. 3a and Fig. $3 b$ show that during all the load perturbations the controller presents a smooth response without large overshoots and with a settling time below 3 seconds for both objectives.

In addition, it is shown that the proposed DMPC controller does not affect the performance of the DAPI controller for voltage restoration and normalised reactive power sharing, as shown in Fig. 4a and Fig. 4b, respectively. It is worth noting that there is better regulation of voltage than reactive power sharing. This is because these two objectives are opposed, and more weight was given to the former. 


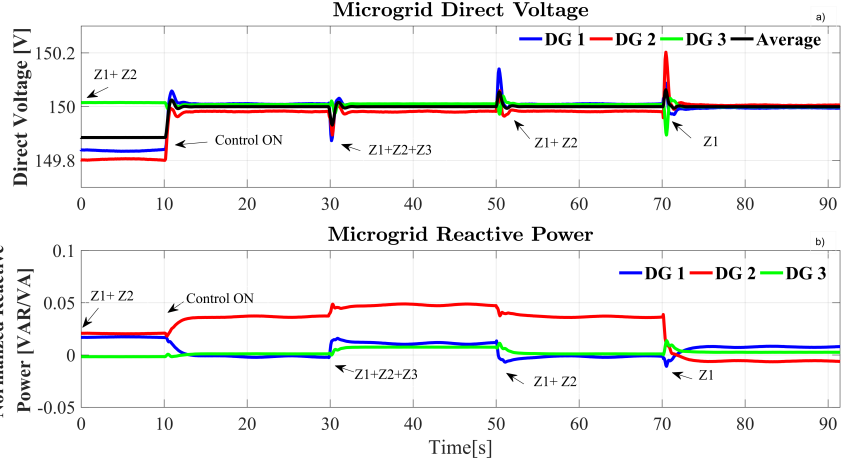

Fig. 4. a) Direct voltage regulation for load changes, b) Reactive power injection for load changes
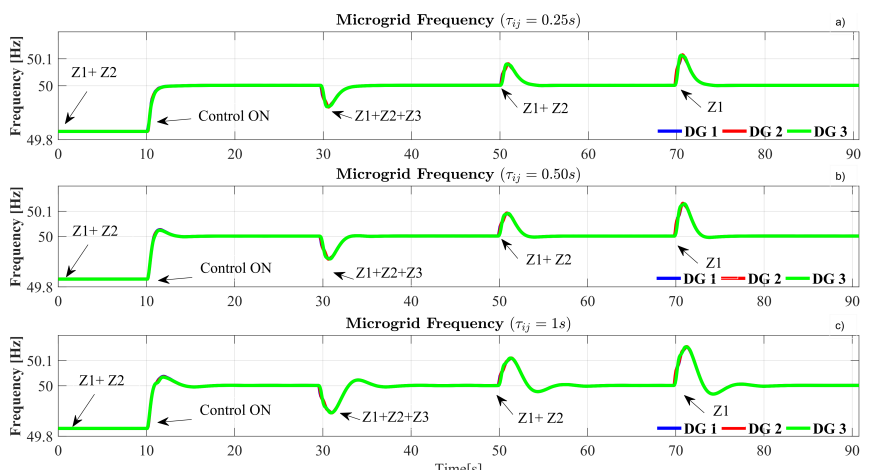

Fig. 5. Frequency regulation in the presence of communication delays a) $\left.\left.\tau_{i j}=0.25 \mathrm{~s}, \mathrm{~b}\right) \tau_{i j}=0.50 \mathrm{~s}, \mathrm{c}\right) \tau_{i j}=1 \mathrm{~s}$

\section{Scenario II - Communication Delay}

This test presents the performance of the proposed controller when there is a constant delay $\left(\tau_{i j}\right)$ in all links of the communication network, whilst the estimated delay $\left(\hat{\tau}_{i j}\right)$ is kept constant at one sample, as shown in (9a) and (12). For each test, the same load perturbations of scenario $I$ are applied. Three cases were considered: a) small time-delay $\left(\tau_{i j}=0.25 \mathrm{~s}\right)$ b) intermediate time-delay $\left(\tau_{i j}=0.5 \mathrm{~s}\right)$ and c) large time-delay $\left(\tau_{i j}=1 s\right)$. Note that the worst-case scenario represents a delay of 20 samples, which is four times the prediction horizon $N_{y}$.

The frequency restoration is the most affected variable under the presence of delays. Figures Fig. 5a, Fig. 5b and Fig. $5 \mathrm{c}$ show the frequency of each DG for the cases of small time-delay $\left(\tau_{i j}=0.25 \mathrm{~s}\right)$, intermediate time-delay $\left(\tau_{i j}=0.5 \mathrm{~s}\right)$, and large time-delay $\left(\tau_{i j}=1 \mathrm{~s}\right)$, respectively. It is observed that the larger the time-delay the larger the overshoot and settling time. However, these two parameters are still small, even for the worse case-scenario, i.e. for $\left(\tau_{i j}=1 \mathrm{~s}\right)$ the overshoot is negligible (less than $0.3 \%$ ) and the settling time is below ten seconds.

The economic real power dispatch for the same three timedelays is presented in Fig. 6. It is observed that the settling time is practically unaffected; even in the worst case $\left(\tau_{i j}=1 \mathrm{~s}\right)$, the settling time is lower than five seconds. The overshoot slightly increases, as the time-delay increases. This is seen most when the controller is activated; nevertheless, it is still negligible. From these results, it is possible to establish that the DMPC is robust against communication delays over and above the prediction horizon. This is because the MPC uses

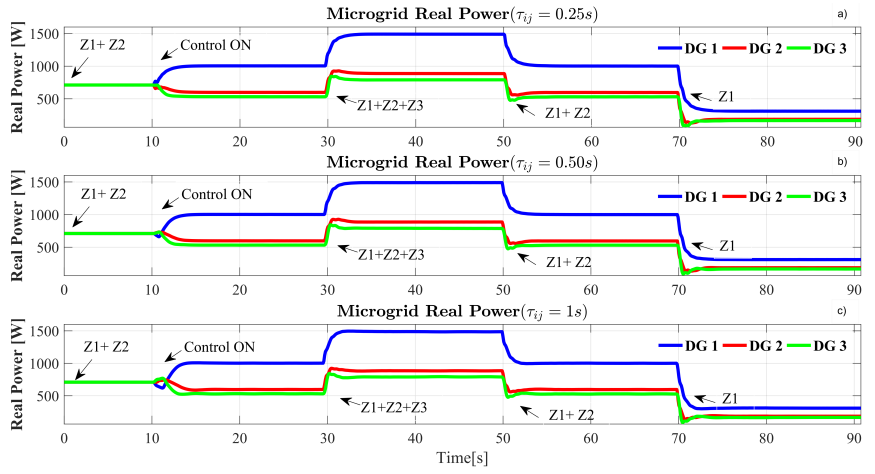

Fig. 6. Real power injection in the presence of communication delays a) $\left.\left.\tau_{i j}=0.25 \mathrm{~s}, \mathrm{~b}\right) \tau_{i j}=0.50 \mathrm{~s}, \mathrm{c}\right) \tau_{i j}=1 \mathrm{~s}$

the rolling horizon property, which determines the appropriate control sequence even with past information from neighbouring DGs.

\section{E. Scenario III - Communication Link Failure}

To analyse the performance of the controller against communication link failures, the following test was carried out. The test begins with two loads connected at different nodes $\left(Z_{1}\right.$ and $\left.Z_{2}\right)$. At $t=10 \mathrm{~s}$, the controller is enabled. A communication failure is forced at $t=30 \mathrm{~s}$ between DG1 and DG2, so the adjacency matrix is modified as shown in (19), and the control algorithm identifies automatically the failure by calculating (9a) and (12) only with the information received. $Z_{3}$ is connected at $t=50 \mathrm{~s}$ and disconnected at $t=70 \mathrm{~s}$. Finally, the communication link is restored at $t=90 \mathrm{~s}$.

$$
A(k)=\left[\begin{array}{lll}
a_{11} & a_{12} & a_{13} \\
a_{21} & a_{22} & a_{23} \\
a_{31} & a_{32} & a_{33}
\end{array}\right]=\left[\begin{array}{ccc}
0 & 0 & 1 \\
0 & 0 & 1 \\
1 & 1 & 0
\end{array}\right]
$$

The results are shown in Fig. 7a and Fig. 7b. It is observed that the controller performance is not impaired, and the control objectives are achieved. Therefore, the control strategy is robust against communication failures. Nevertheless, the transient response is different, specifically, the settling time is increased to nearly ten seconds. This is because the communication matrix (A) is not complete (19), and the control objectives are directly related to known information from the neighbouring DGs.

\section{F. Scenario IV - Plug and Play}

This test presents the controller's response when an unscheduled failure occurs in a specific DG. The test starts with two loads connected at $t=0 \mathrm{~s}\left(Z_{1}\right.$ and $\left.Z_{2}\right)$ and the controller is enabled and at $t=10 \mathrm{~s}$, where the adjacency matrix is represented by (18). At $t=30 \mathrm{~s}$ DG2 is taken out of service, i.e. DG2 is disconnected from both the electrical system and the communication network. Thus, the adjacency matrix is modified as shown in Fig. 8a. The MG continues operating with DG1 and DG3 connected. Next at $t=50 \mathrm{~s}$, the total load is connected. At $t=70 \mathrm{~s}$, after a synchronisation routine, DG2 is reconnected to the MG. Finally, $Z_{3}$ is disconnected.

Note that although DG2 is disconnected from the MG, it is not turned off. Only its secondary control is disabled, but 

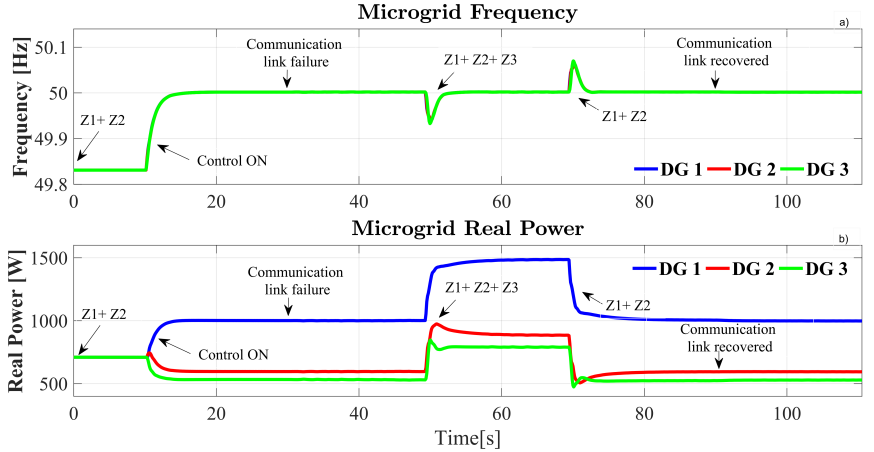

Fig. 7. a) Frequency regulation in the presence of communication failure between DG1 and DG2, b) Real power injection against communication failure between DG1 and DG2
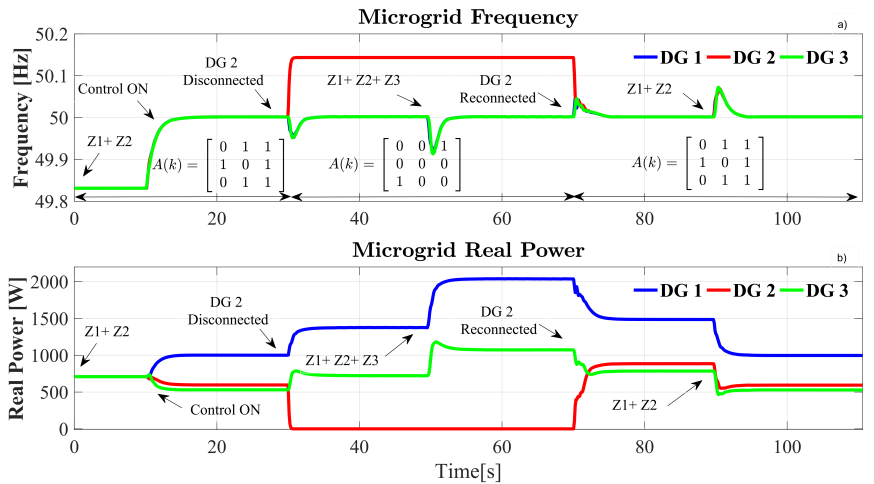

Fig. 8. Plug and Play test - a) Frequency regulation, b) Real power injection

its primary control continues operating. When DG2 is disconnected or reconnected the adjacency matrix is updated, and the remaining controllers identify this failure by calculating (9a) and (12) only with the information received. Therefore, the remaining DG units optimise the consensus terms with only the operating units.

Fig. 8a presents the frequency restoration. It is observed that the operating DGs restore the frequency adequately during the whole test without overshoots or long settling times. Similarly, Fig. $8 \mathrm{~b}$ presents the real power contribution. It is observed that when DG2 is disconnected although both DG units increase their contributions, DG1 takes the majority of the load; however, its maximum power capacity is not exceeded. Therefore, the proposed controller has a Plug-and-Play capability and always respects the physical power capacity of the DGs.

\section{COMPARISON Against DMPC Without ECONOMIC DISPATCH}

In this section, a comparison of our proposal and the work of [12] is made using simulation studies. The objectives of [12] are to restore frequency and voltage while ensuring proportional power sharing. Therefore, it shares real power without considering generation costs.

Both control strategies are tested for a load change scenario, and their adjacency matrices are expressed in (18). For both controllers, the test is described as follows. The test starts with the controller turned on and $Z_{1}$ and $Z_{2}$ connected. Then at $t=20 \mathrm{~s}$ and $t=40 \mathrm{~s} Z_{3}$ is connected and disconnected, respectively. Finally, at $\mathrm{t}=60 \mathrm{~s} Z_{1}$ is disconnected. The results for the real power dispatch of both proposals are presented

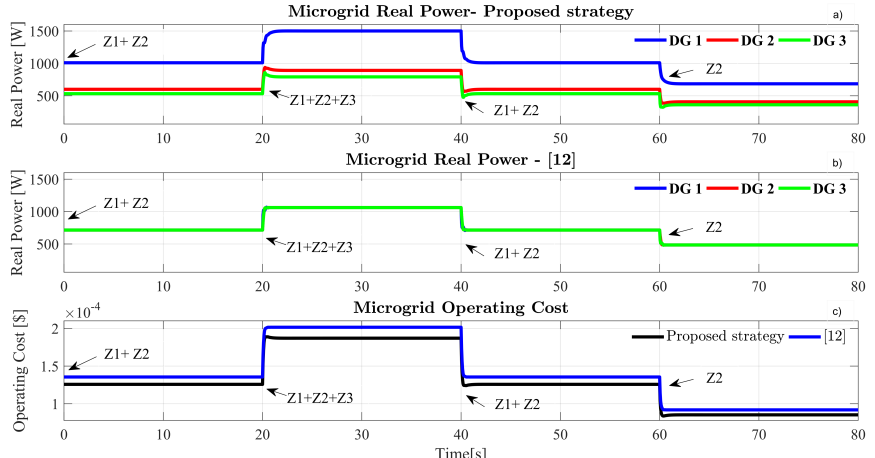

Fig. 9. Operation comparison- a) Proposed strategy - real power injection, b) [12] - real power injection, c) Operation costs comparison

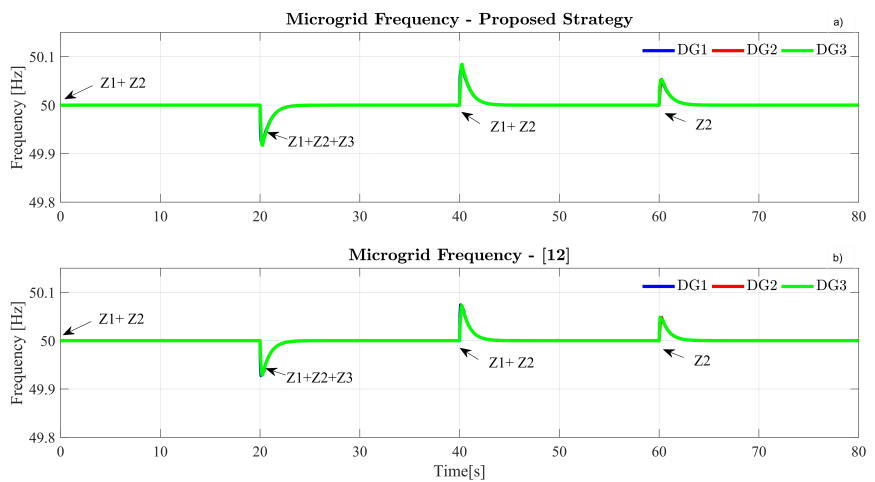

Fig. 10. Frequency regulation comparison- a) Proposed strategy, b) [12]

in Fig. 9a and Fig. 9b, respectively. Conversely, Fig. 9c presents a comparison between their operation costs, which were obtained evaluating the DGs' power contribution in (4a) with the coefficients of Table IV. It is observed that our proposal has a lower economic operation during the whole test. Furthermore, the frequency regulation response for both approaches is presented in Fig. 10, where both approaches have a pretty similar response. This is because both strategies seek the frequency regulation objective and include the $P-\omega$ droop model as a constraint.

The operating costs of the complete test, savings and optimisation time for both techniques are presented in Table V. The cost saving is calculated by Cost $_{\text {saving }}=\frac{\text { Cost }_{2}-\text { cost }_{1}}{\text { Cost }_{2}} \cdot 100 \%$ [22], where Cost 1 is the operation cost of the proposed strategy and $\mathrm{Cost}_{2}$ is the operation cost of [12], respectively. The optimisation time is reduced because the proposed DMPC is simplified, specifically to tackle the objectives of economic dispatch and frequency restoration. Furthermore, note that as the proposed strategy uses the same typical measurements of the primary control and a voltage observer to estimate the voltage after the coupling inductance $\left(\hat{V}_{i}^{B}\right)$, its implementation cost is reduced, and it does not require physical changes at the electrical level. On the other hand, to implement the work of [12] additional three-phase sensors are needed to measure $\left(V_{i}^{B}\right)$ as well as fibre optical cables to communicate these measurements to the controllers.

\section{CONCLUSIONS AND FinAl REMARKS}

This paper presents a novel distributed predictive control strategy to cope with optimal dispatch and frequency reg- 
TABLE V

CONTROLLERS' MAIN DIFFERENCES

\begin{tabular}{l|l|l}
\hline \hline Control strategy & Proposed strategy & {$[12]$} \\
\hline Operation cost [USç] & 0.0105 & 0.0113 \\
\hline Cost $_{\text {saving }}[\%]$ & 7.2 & 0 \\
\hline Optimisation time [s] & $4.8 \mathrm{E}-3$ & $5.5 \mathrm{E}-3$ \\
\hline Require additional sensors & No & Yes \\
\hline \hline
\end{tabular}

ulation for isolated microgrids. The proposed controller is able to maintain frequency regulation and economic dispatch at the same, respecting the maximum power limits of each DG. The dynamic performance of the controller is evaluated experimentally and discussed under four test scenarios. The controller effectively tackles communication link issues and the connection/disconnection of DGs. Future research will investigate the application of the control strategy to hybrid $\mathrm{ac} / \mathrm{dc}$ microgrids and the inclusion of voltage/reactive power control in the proposed predictive control strategy.

\section{APPENDIX A}

\section{REDUCED-ORDER NON-LINEAR OBSERVER TO ESTIMATE THE VOLTAGE AFTER A COUPLING INDUCTANCE}

In this appendix, we provide an explanation of the application of the reduced-order non-linear observer proposed in [28] to estimate the voltage $\left(\hat{V}_{i}^{B}\right)$ after the coupling inductance $\left(L_{i}\right)$. For a complete description of the demonstration of the observer, the reader is encouraged to read the aforementioned work.

Consider a class of nonlinear system given by

$$
\dot{\mathbf{x}}=\mathbf{F}\left(\mathbf{x}_{a}, \mathbf{u}\right) \mathbf{x}+\mathbf{g}\left(\mathbf{x}_{a}, \mathbf{u}\right)
$$

where $\mathbf{x} \in \mathbb{R}^{n \times 1}$ is the state vector and $\mathbf{u} \in \mathbb{R}^{m \times 1}$ is the input vector, with $\mathbf{F} \in \mathbb{R}^{n \times n}$ and $\mathrm{g} \in \mathbb{R}^{n \times 1}$. The state vector can be partionated as $\mathbf{x}=\left[\mathbf{x}_{a} \mathbf{x}_{b}\right]^{T}$, where $\mathbf{x}_{a} \in \mathbb{R}^{n_{a} \times 1}$ contains measurable variables and $\mathbf{x}_{b} \in \mathbb{R}^{n_{b} \times 1}$ contains non-measurable variables. The representation of (20) can be rewritten as follows.

$$
\left[\begin{array}{c}
\dot{\mathbf{x}}_{a} \\
\dot{\mathbf{x}}_{b}
\end{array}\right]=\left[\begin{array}{ll}
\mathbf{N}\left(\mathbf{x}_{a}, \mathbf{u}\right) & \mathbf{M}\left(\mathbf{x}_{a}, \mathbf{u}\right) \\
\mathbf{R}\left(\mathbf{x}_{a}, \mathbf{u}\right) & \mathbf{S}\left(\mathbf{x}_{a}, \mathbf{u}\right)
\end{array}\right]\left[\begin{array}{l}
\mathbf{x}_{a} \\
\mathbf{x}_{b}
\end{array}\right]+\left[\begin{array}{l}
\mathbf{g}_{a}\left(\mathbf{x}_{a}, \mathbf{u}\right) \\
\mathbf{g}_{b}\left(\mathbf{x}_{a}, \mathbf{u}\right)
\end{array}\right]
$$

The previously described non-linear observer of reducedorder is used to estimate the voltage $\left(\hat{V}_{i}^{B}\right)$ after the coupling inductance $\left(L_{i}\right)$, as shown at the bottom of Fig. 1. The observer works in the $\alpha-\beta$ framework. Considering $\hat{V}_{\alpha, i}^{B}=V_{m} \sin \left(\theta_{i}\right)$ and $\hat{V}_{\beta, i}^{B}=V_{m} \cos \left(\theta_{i}\right)$, where $V_{m}$ depends on the $a b c-\alpha \beta$ transformation used. Equation (22), which represents the estimated states, is obtained deriving both expressions. Where $\omega_{o}$ is the nominal frequency.

$$
\begin{gathered}
\hat{\dot{V}}_{\alpha, i}^{B}=V_{m} \omega_{o} \cos \left(\theta_{i}\right)=\omega_{o} \hat{V}_{\beta, i}^{B} \\
\dot{\hat{V}}_{\beta, i}^{B}=-V_{m} \omega_{o} \sin \left(\theta_{i}\right)=-\omega_{o} \hat{V}_{\alpha, i}^{B}
\end{gathered}
$$

Equation (23), which represents the measured estates, is obtained applying the Kirchhoff's voltage law to the circuit of Fig. 1. Where $R_{i}$ represents the cable resistance, and is assumed as $R_{i}=0.01 \Omega$.

$$
\begin{aligned}
& L_{i} \dot{i}_{\alpha, i}=-R i_{\alpha, i}+V_{\alpha, i}-\hat{V} \alpha, i^{B} \\
& L_{i} \dot{i}_{\beta, i}=-R i_{\beta, i}+V_{\beta, i}-\hat{V} \beta, i^{B}
\end{aligned}
$$

The measured states $\left(x_{a}\right)$ and estimated states $\left(x_{b}\right)$ are presented in Equation (24). These are obtained expressing (22) and (23) in the required form of the observer (21). Where its inputs are the measured values of $V_{i}$ and $i_{i}$ (at the output of the LC filter), both in the $\alpha-\beta$ framework.

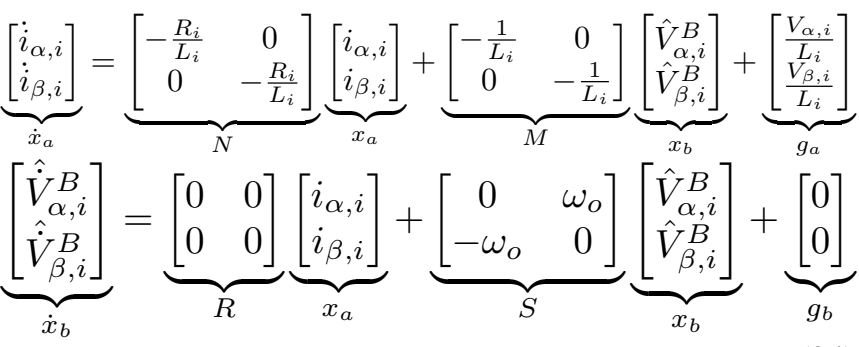

The structure of the observer is presented in (25).

$$
\begin{gathered}
\dot{\xi}=\mathrm{A}_{r}(\xi+\mathrm{Gw})+\mathrm{B}_{r} \\
\hat{\mathbf{x}}_{b}=\xi+\mathrm{Gw}
\end{gathered}
$$

Where $\mathbf{w}$ is a transformation that depends on the measured variables to obtain a linear dynamic of the error.

$$
\mathbf{w}=\mathbf{T}\left(\mathbf{x}_{\mathbf{a}}\right)=\left[\begin{array}{l}
\omega_{1} \\
\omega_{2}
\end{array}\right]=\left[\begin{array}{l}
-L_{i}\left(i_{\alpha, i}+\frac{\omega_{o} i_{\beta, i}}{g v}\right) \\
-L_{i}\left(i_{\beta, i}-\frac{\omega_{o} i_{\alpha, i}}{g v}\right)
\end{array}\right]
$$

The estimation error dynamic $\mathbf{A}_{r}$ (27) is obtained through pole placement so that the observer is able to follow the phase of the estimated voltages, and it is faster than the secondary controller. Finally, the gains $g v$ were placed at -31500 , and $\mathbf{B}_{r}$ is represented in (28).

$$
\begin{gathered}
\mathbf{A}_{r}=-\mathbf{G}=\left[\begin{array}{cc}
-g v & 0 \\
0 & -g v
\end{array}\right] \\
\mathbf{B}_{r}=\mathbf{R x}_{a}+\mathbf{g}_{b}-\mathbf{G} \frac{\partial \mathbf{T}}{\partial \mathbf{x}_{a}}\left(\mathbf{N x}_{a}+\mathbf{g}_{a}\right)
\end{gathered}
$$

\section{REFERENCES}

[1] Department of Energy office of Electricity Delivery and Energy Reliability, "Summary report : 2012 DOE microgrid workshop," U.S. Dep. Energy, Tech. Rep., Jul. 2012.

[2] J. Vásquez, J. Guerrero, M. Savaghebi, E. G. Carrasco, and R. Teodorescu, "Modeling, analysis, and design of stationary reference frame droop controlled parallel three-phase voltage source inverters," IEEE Trans. Ind. Electron., vol. 60, pp. 1271-1280, Apr. 2013.

[3] D. E. Olivares, A. Mehrizi-Sani, A. H. Etemadi, C. A. Cañizares, R. Iravani, M. Kazerani, A. H. Hajimiragha, O. Gomis-Bellmunt, M. Saeedifard, R. Palma-Behnke, G. A. Jiménez-Estévez, and N. D. Hatziargyriou, "Trends in microgrid control," IEEE Trans. Smart Grid, vol. 5, no. 4, pp. 1905-1919, Jul. 2014.

[4] J. M. Guerrero, J. C. Vásquez, J. Matas, L. G. de Vicuna, and M. Castilla, "Hierarchical control of droop-controlled ac and de microgrids-a general approach toward standardization," IEEE Trans. Ind. Electron., vol. 58, no. 1, pp. 158-172, Jan. 2011.

[5] S. K. Sahoo, A. K. Sinha, and N. K. Kishore, "Control techniques in AC, DC, and hybrid AC-DC microgrid: A review," IEEE Trans. Emerg. Sel. Topics Power Electron., vol. 6, no. 2, pp. 738-759, Jun. 2018.

[6] A. Bidram and A. Davoudi, "Hierarchical structure of microgrids control system," IEEE Trans. Smart Grid, vol. 3, no. 4, pp. 1963-1976, Dec. 2012.

[7] F. Dörfler, J. W. Simpson-Porco, and F. Bullo, "Breaking the hierarchy: Distributed control and economic optimality in microgrids," IEEE Trans. Control Netw. Syst., vol. 3, no. 3, pp. 241-253, Sep. 2016. 
[8] Z. Li, Z. Cheng, J. Liang, J. Si, L. Dong, and S. Li, "Distributed event-triggered secondary control for economic dispatch and frequency restoration control of droop-controlled AC microgrids," IEEE Trans. Sustain. Energy, vol. 11, no. 3, pp. 1938-1950, Jul. 2020.

[9] X. Feng, A. Shekhar, F. Yang, R. E. Hebner, and P. Bauer, "Comparison of hierarchical control and distributed control for microgrid," Electr. Power Components Syst., vol. 45, no. 10, pp. 1043-1056, Jul. 2017.

[10] J. W. Simpson-Porco, Q. Shafiee, F. Dorfler, J. C. Vasquez, J. M. Guerrero, and F. Bullo, "Secondary frequency and voltage control of islanded microgrids via distributed averaging," IEEE Trans. Ind. Electron., vol. 62, no. 11, pp. 7025-7038, Nov. 2015.

[11] Z. Guo, S. Li, and Y. Zheng, "Feedback linearization based distributed model predictive control for secondary control of islanded microgrid," Asian J. Control, vol. 22, no. 1, pp. 460-473, Jan. 2020.

[12] J. S. Gómez, D. Sáez, J. W. Simpson-Porco, and R. Cardenas, "Distributed predictive control for frequency and voltage regulation in microgrids," IEEE Trans. Smart Grid, vol. 11, no. 2, pp. 1319-1329, Mar. 2020.

[13] S. V. Viscido, J. K. Parrish, and D. Grünbaum, "The effect of population size and number of influential neighbors on the emergent properties of fish schools," Ecol. Modell., vol. 183, no. 2, pp. 347 - 363, Apr. 2005.

[14] F. Guo, C. Wen, J. Mao, and Y. Song, "Distributed secondary voltage and frequency restoration control of droop-controlled inverter-based microgrids," IEEE Trans. Ind. Electron., vol. 62, no. 7, pp. 4355-4364, Jul. 2015.

[15] B. Wei, Y. Gui, S. Trujillo, J. M. Guerrero, and J. C. Vásquez, "Distributed average integral secondary control for modular ups systemsbased microgrids," IEEE Trans. Power Electron., vol. 34, no. 7, pp. 6922-6936, Jul. 2019.

[16] G. Lou, W. Gu, Y. Xu, M. Cheng, and W. Liu, "Distributed mpc-based secondary voltage control scheme for autonomous droop-controlled microgrids," IEEE Trans. Sustain. Energy, vol. 8, no. 2, pp. 792-804, Apr. 2017.

[17] K. Liu, T. Liu, Z. Tang, and D. J. Hill, "Distributed MPC-based frequency control in networked microgrids with voltage constraints," IEEE Trans. Smart Grid, vol. 10, no. 6, pp. 6343-6354, Nov. 2019

[18] Y. Zheng, S. Li, and R. Tan, "Distributed model predictive control for on-connected microgrid power management," IEEE Trans. Control Syst. Technol., vol. 26, no. 3, pp. 1028-1039, May 2018.

[19] Z. Guo, H. Jiang, Y. Zheng, and S. Li, "Distributed model predictive control for efficient operation of islanded microgrid," in 2017 Chinese Automation Congress $(C A C)$. Institute of Electrical and Electronics Engineers Inc., Oct. 2017, pp. 6253-6258.

[20] Y. Han, K. Zhang, H. Li, E. A. A. Coelho, and J. M. Guerrero, "Masbased distributed coordinated control and optimization in microgrid and microgrid clusters: A comprehensive overview," IEEE Trans. Power Electron., vol. 33, no. 8, pp. 6488-6508, Aug. 2018.

[21] J. Llanos, D. E. Olivares, J. W. Simpson-Porco, M. Kazerani, and D. Saez, "A novel distributed control strategy for optimal dispatch of isolated microgrids considering congestion," IEEE Trans. Smart Grid, vol. 10 , no. 6, pp. 6595-6606, Nov. 2019.

[22] G. Chen and Z. Guo, "Distributed secondary and optimal active power sharing control for islanded microgrids with communication delays," IEEE Trans. Smart Grid, vol. 10, no. 2, pp. 2002-2014, Mar. 2019.

[23] C. Bordons, F. Garcia-Torres, and M. A. Ridao, Model predictive control of microgrids, 1st ed., ser. Advances in Industrial Control. Springer International Publishing, 2020.

[24] B. Huang, L. Liu, Y. Li, and H. Zhang, "Distributed optimal energy management for microgrids in the presence of time-varying communication delays," IEEE Access, vol. 7, pp. 83 702-83 712, Jun. 2019.

[25] M. Yazdanian and A. Mehrizi-Sani, "Distributed control techniques in microgrids," IEEE Trans. Smart Grid, vol. 5, no. 6, pp. 2901-2909, Nov. 2014.

[26] S. Boyd and L. Vandenberghe, Convex optimization. USA: Cambridge University Press, 2004.

[27] R. Wai, Q. Zhang, and Y. Wang, "A novel voltage stabilization and power sharing control method based on virtual complex impedance for an off-grid microgrid," IEEE Trans. Power Electron., vol. 34, no. 2, pp. 1863-1880, Feb. 2019.

[28] P. Kundur, Power system stability and control. McGraw-Hill, 1994, vol. 7.

[29] A. E. Leon and J. A. Solsona, "Design of reduced-order nonlinear observers for energy conversion applications," IET Control Theory Appl., vol. 4, no. 5, pp. 724-734, May 2010.

[30] W. Ren, R. W. Beard, and E. M. Atkins, "Information consensus in multivehicle cooperative control," IEEE Control Syst., vol. 27, no. 2, pp. 71-82, Jul. 2007.
[31] E. F. Camacho and C. Bordons, "Constrained model predictive control," in Model Predictive Control, 2nd ed., ser. Advanced Textbooks in Control and Signal Processing. London: Springer London, 2007.

[32] C. Schmid and L. T. Biegler, "Quadratic programming methods for reduced hessian sqp," Comput. Chem. Eng., vol. 18, no. 9, pp. 817832, Sep. 1994.

[33] K. J. Åström and B. Wittenmark, Adaptive Control. Addison-Wesley, 1989.

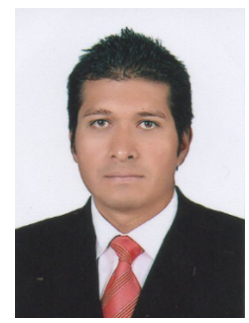

Alex Navas F. (S'18) was born in Latacunga, Ecuador. He received the B.Sc. degree in electronic engineering from the Army Polytechnic School ESPE, Ecuador, in 2015. He is currently pursuing the double $\mathrm{PhD}$ degree in electrical engineering with the University of Chile, Chile, and the University of Nottingham, United Kingdom. His research interests include the control and management of microgrids, model predictive control applied to microgrids and renewable energies.

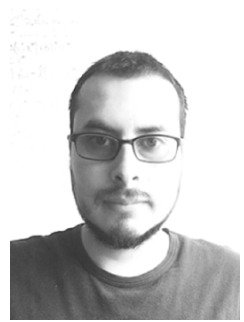

Juan S. Gómez was born in Bogotá, Colombia He received the B.E degree in Electronics Engineering from "Universidad Distrital- Francisco José de Caldas", Bogotá, Colombia in 2011, and the Ph.D. degree in Electrical Engineering from the University of Chile (Universidad de Chile), Santiago, Chile in 2020. Dr. Gómez is currently affiliated as postdoctoral researcher on microgrids applications for mining industry at the Pontificia Universidad Católica de Chile (Anillo project ACT192013). He previously worked in the Colombian oil and gas industry as a Project Engineer and as a Specialist Automation Engineer between 2010 and 2016. His research interests are focused mainly on microgrids control, networked control systems, renewable energies, and model-based predictive control. extractive metallurgy.

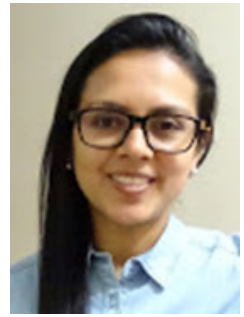

Jacqueline Llanos (S'10-M'13) was born in Latacunga, Ecuador. She received the B.Sc. and Engineer degrees in electronic engineering from the Army Polytechnic School, Ecuador. She received the M.Sc. and Ph.D. degrees in electrical engineering from the University of Chile, Santiago. She is currently an Assistant Professor with the Department of Electrical and Electronic, Universidad de las Fuerzas Armadas ESPE, Ecuador. Her current research interests include control and management of microgrids, control of power generation plants, distributed control and predictive control.

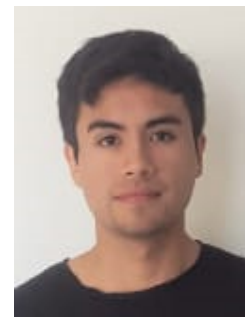

Erwin Rute was born in Puerto Montt, Chile. He received the B.Sc. degree in electrical engineering from University of Chile, Chile, in 2020. Also, he received the M.Sc. degree in electrical engineering from the University of Chile, Chile, in 2020. His research interests include the control of microgrids and model predictive control applied to microgrids. 


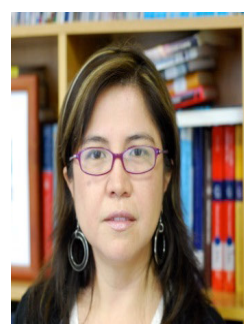

Doris Sáez (S'93-M'96-SM'05) was born in Panguipulli, Chile. She received the M.Sc. and Ph.D. degrees in electrical engineering from the Pontificia Universidad Católica de Chile, Santiago, Chile, in 1995 and 2000, respectively. She is currently a Full Professor with the Department of Electrical Engineering and the Head of the Indigenous People Program, Faculty of Mathematical and Physical Sciences, University of Chile, Santiago. She has coauthored the books Hybrid Predictive Control for Dynamic Transport Problems (Springer Verlag, 2013) and Optimization of Industrial Processes at Supervisory Level: Application to Control of Thermal Power Plants (Springer-Verlag, 2002). Her research interests include predictive control, fuzzy control design, fuzzy identification, and control of microgrids. She also serves as an Associate Editor for the IEEE Transactions on Smart Grid.

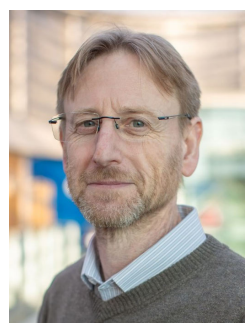

Mark Sumner (SM'05) received the B.Eng. degree in electrical and electronic engineering from the University of Leeds, in 1986, and the Ph.D. degree in induction motor drives from the University of Nottingham, U.K., in 1990. He was with Rolls Royce Ltd., U.K. He was also a Research Assistant and a Lecturer in October 1992. He is currently a Professor of electrical energy systems with the Power Electronics, Machines and Control Group (PEMC Group), University of Nottingham. His research interests include control of power electronic systems, including sensorless motor drives, diagnostics and prognostics for drive systems, power electronics for enhanced power quality, and novel power system fault location strategies. 